\title{
Irreducible decomposition of strain gradient tensor in isotropic strain gradient elasticity
}

\author{
Markus Lazar* \\ Heisenberg Research Group, \\ Department of Physics, \\ Darmstadt University of Technology, \\ Hochschulstr. 6, \\ D-64289 Darmstadt, Germany
}

April 26, 2016

\begin{abstract}
In isotropic strain gradient elasticity, we decompose the strain gradient tensor into its irreducible pieces under the $n$-dimensional orthogonal group $O(n)$. Using the Young tableau method for traceless tensors, four irreducible pieces $(n>2)$, which are canonical, are obtained. In three dimensions, the strain gradient tensor can be decomposed into four irreducible pieces with $7+5+3+3$ independent components whereas in two dimensions, the strain gradient tensor can be decomposed into three irreducible pieces with $2+2+2$ independent components. The knowledge of these irreducible pieces is extremely useful when setting up constitutive relations and strain energy.
\end{abstract}

Keywords: strain gradient elasticity, irreducible decomposition, Young tableaux, traceless tensors, isotropy

\section{Introduction}

Irreducible tensors are of fundamental interest in the representation theory of groups and are important in applied mathematics and theoretical physics (e.g., 32, 33, 13, 3] ). Irreducible tensors of rank three and four are used in differential geometry and metric affine gauge theory (e.g., [14]). In high energy physics, tensors of arbitrary rank being irreducible with respect to the Lorentz group $\mathrm{SO}(1,3)$ are necessary (e.g., [10, 11]). In generalized elasticity, Toupin [30] discussed that general tensors of rank three have four irreducible symmetry parts. Toupin [30] used four symmetry operators, which correspond to the four relevant Young symmetries (symmetries of Young tableaux, see below). The four irreducible symmetry parts discussed by Toupin [30] are irreducible with respect to the $n$-dimensional general linear group $G L(n)$. For couple-stress elasticity, only two irreducible symmetry parts survive since the couple-stress tensor is antisymmetric in two indices. Currently, there is a renewed interest in irreducible tensors in elasticity theory (e.g., [15]) and in generalized elasticity theory, especially in gradient elasticity theory (e.g., [1, 2, 12]).

*E-mail address: lazar@fkp.tu-darmstadt.de (M. Lazar). 
Mindlin's theory of strain gradient elasticity [23] is a well-suited framework to model the behavior of elastic materials up to the nano-scale. Using ab initio calculations, Shodja et al. 29] found that the characteristic length scale parameters of Mindlin's gradient elasticity theory are in the order of $\sim 10^{-10} \mathrm{~m}$ for several fcc and bcc materials. Therefore, as a generalization of classical elasticity, gradient elasticity is relevant for nano-mechanical phenomena at such length scales. However, the most general version of Mindlin's strain gradient elasticity has found limited application so far, because of both its complexity and the presence of a large number of new material parameters. The use of irreducible tensors in Mindlin's strain gradient elasticity theory may deliver a better understanding of the general structure of such a theory.

The aim of this work is to give a canonical and unique decomposition of the strain gradient tensor, which is a tensor of rank three, into its irreducible pieces with respect to the orthogonal group $O(n)$ using the method of Young tableaux. Also the connection of the irreducible form of strain gradient elasticity to Mindlin's strain gradient elasticity theory [23] and to strain gradient elasticity theory of Helmholtz type [18, 22] is given.

\section{Prolog}

In strain gradient elasticity (or gradient elasticity of form II), the two state quantities are the elastic strain tensor, which is a symmetric tensor of rank two,

$$
\varepsilon_{i j}=\frac{1}{2}\left(u_{i, j}+u_{j, i}\right), \quad \varepsilon_{i j}=\varepsilon_{j i}
$$

and the elastic strain gradient tensor, which is a tensor of rank three,

$$
\eta_{i j k}:=\varepsilon_{i j, k}, \quad \eta_{i j k}=\eta_{j i k}
$$

is symmetric in the first two indices: $\eta_{i j k} \equiv \eta_{(i j) k}$. Here $u_{i}$ is the displacement vector and a comma denotes the differentiation with respect to the coordinates.

The elastic strain tensor can be decomposed into two irreducible pieces according to

$$
\varepsilon_{i j}={ }^{(1)} \varepsilon_{i j}+{ }^{(2)} \varepsilon_{i j}
$$

with the number of independent components

$$
\frac{n(n+1)}{2}=\frac{(n+2)(n-1)}{2}+1
$$

The irreducible tensor piece ${ }^{(1)} \varepsilon_{i j}$ is defined as symmetric and traceless tensor of rank two, which is the shear tensor

$$
{ }^{(1)} \varepsilon_{i j}=\nexists_{i j}:=\varepsilon_{i j}-\frac{1}{n} \delta_{i j} \varepsilon . ., \quad Z_{. .}=0 .
$$

In tensor analysis and continuum mechanics, this piece is usually called deviator of $\varepsilon_{i j}$ (e.g., [4, 6]). The second irreducible tensor piece ${ }^{(2)} \varepsilon_{i j}$ is the spherical part of $\varepsilon_{i j}$

$$
{ }^{(2)} \varepsilon_{i j}=\frac{1}{n} \delta_{i j} \varepsilon
$$

\footnotetext{
${ }^{1}$ We use the notation of Schouten [27]. Symmetrization over two indices is denoted by parentheses, $A_{(i j)}:=$ $\left(A_{i j}+A_{j i}\right) / 2$ !, antisymmetrization by brackets, $B_{[i j]}:=\left(B_{i j}-B_{j i}\right) / 2$ !. The analogous is valid for more indices, e.g., $C_{(i j k)}:=\left(C_{i j k}+C_{j k i}+C_{k i j}+C_{i k j}+C_{j i k}+C_{k j i}\right) / 3$ !.
} 
with the dilatation $\varepsilon$.

$$
\varepsilon_{. .}:=\varepsilon_{i i}=\delta_{i j} \varepsilon_{i j}
$$

and $\delta_{i i}=n$.

\section{$3 \quad$ Irreducible decomposition of the strain gradient tensor $\eta_{i j k}$}

\subsection{Basic remarks}

Let $O(n)$ be the orthogonal group in $n$-dimensions. $O(n)$-irreducible tensors are traceless tensors having defined symmetry classes associated with so-called Young tableaux (see Appendix $\mathrm{A}$ and [13, 33, 5]). Starting from the subspace of traceless tensors and applying the Young symmetry to obtain irreducible tensors of a given symmetry type. However, not all Young tableaux are admissible. The traceless tensors corresponding to Young tableaux where the sum of the lengths of the first two columns exceeds $n$ must be identically zero [13]. Thus, only the Young tableaux are permissible for which the sum of the lengths of the first two columns is: $\mu_{1}+\mu_{2} \leq n$. Two tableaux $T$ and $T^{\prime}$ (and the corresponding tensor representations) for which the first columns are related by $\mu_{1}^{\prime}=n-\mu_{1}$, where $\mu_{1} \leq n / 2$, are called associated. If $\mu_{1}=\mu_{1}^{\prime}=n / 2$ ( $n$ is even), then $T$ and $T^{\prime}$ are called self-associated. If we consider the special (or proper) orthogonal group $S O(n) \subset O(n)$, for which $\boldsymbol{a} \in S O(n)$ : $\operatorname{det} \boldsymbol{a}=+1$, the representations corresponding to the associated Young pattern $T$ and $T^{\prime}$ become equivalent, whereas representations corresponding to self-associated pattern decompose into two nonequivalent irreducible representations. As an example for $n=3$, the pattern $T=(1)$ and $T^{\prime}=(1,1)$ are associated:

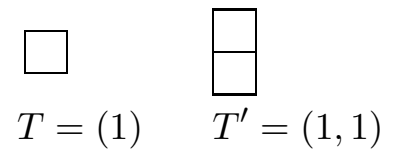

The pattern $T=(1)$ describes vectors, e.g. $V_{i}$, while $T^{\prime}=(1,1)$ describes antisymmetric tensors of rank two, e.g. $T_{[j k]}$. The vector and axial vector representations $V_{i}$ and $A_{i}=$ $\frac{1}{2} \epsilon_{i j k} T_{[j k]}$, respectively, are associated ones ( $\epsilon_{i j k}$ is the three-dimensional Levi-Cività tensor). Under $S O(3)$ both types transform in the same way and $T$ and $T^{\prime}$ are equivalent. Under $O(3)$, the tensors for $T=(1)$ change sign (polar vector), whereas $T^{\prime}=(1,1)$ do not (axial vector).

\subsection{Young symmetries of a general tensor of rank three}

For a general tensor of rank three, $F_{i j k}$, there are four non-vanishing standard Young tableaux if $n>2$, namely

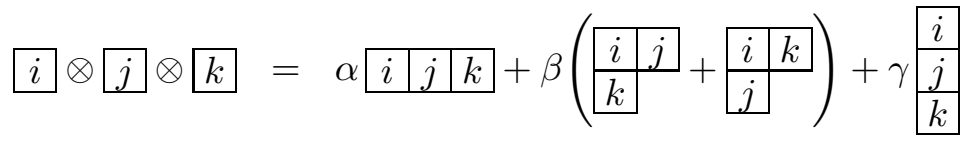

where the coefficients $\alpha, \beta, \gamma$ are determined by the formula (A.6) and they are (see also [13])

$$
\alpha=\frac{f_{[3]}}{3 !}=\frac{1}{3 !}, \quad \beta=\frac{f_{[2,1]}}{3 !}=\frac{2}{3 !}, \quad \gamma=\frac{f_{[1,1,1]}}{3 !}=\frac{1}{3 !} .
$$


Thus, for a general tensor of rank three $F_{i j k}$, the Young tableaux in (9) with (10) give the following tensor decomposition

$$
F_{i j k}={ }_{\mathrm{S}} F_{i j k}+{ }_{\overline{\mathrm{P}}} F_{i j k}+{ }_{\mathrm{P}} F_{i j k}+{ }_{\mathrm{A}} F_{i j k}
$$

where

$$
\begin{aligned}
{ }_{\mathrm{S}} F_{i j k} & =\frac{1}{6}\left(F_{i j k}+F_{j k i}+F_{k i j}+F_{i k j}+F_{j i k}+F_{k j i}\right) \\
{ }_{\mathrm{A}} F_{i j k} & =\frac{1}{6}\left(F_{i j k}+F_{j k i}+F_{k i j}-F_{i k j}-F_{j i k}-F_{k j i}\right) \\
\mathrm{P}_{\mathrm{P}} F_{i j k} & =\frac{1}{3}\left(F_{i j k}+F_{j i k}-F_{k j i}-F_{j k i}\right) \\
{ }_{\mathrm{P}} F_{i j k} & =\frac{1}{3}\left(F_{i j k}+F_{k j i}-F_{j i k}-F_{k i j}\right) .
\end{aligned}
$$

The four tensor pieces (12)-(15) are irreducible with respect to the $n$-dimensional general linear group $G L(n)(n>2)$. The tensor ${ }_{S} F_{i j k}$ is a completely symmetric tensor of rank three corresponding to the pattern (3), ${ }_{\mathrm{A}} F_{i j k}$ is a completely antisymmetric tensor of rank three corresponding to the pattern $(1,1,1)$. Since there are two standard tableaux for the pattern $(2,1)$, two irreducible tensors exist for this pattern, namely ${ }_{\overline{\mathrm{P}}} F_{i j k}$ which is symmetric in $i$ and $j$ and antisymmetric in $i$ and $k$, and ${ }_{\mathrm{P}} F_{i j k}$ which is symmetric in $i$ and $k$ and antisymmetric in $i$ and $j$. 2 Note that the tensor pieces (12), (14), (15) are reducible with respect to the group $O(n)$, since they are not traceless; only the totally antisymmetric piece (13) is already irreducible with respect to the group $O(n)$, since it is traceless by construction. The irreducible decomposition of the general tensor $F_{i j k}$ with respect to $O(n)$ is given in Appendix E,

The tensor pieces ${ }_{\mathrm{S}} F_{i j k}$ and ${ }_{\mathrm{P}} F_{i j k}$ are the relevant ones in strain gradient elasticity (see below), whereas the pieces ${ }_{\mathrm{A}} F_{i j k}$ and ${ }_{\mathrm{P}} F_{i \underline{i k}}$ are the relevant ones in couple-stress elasticity (see [30]), in dislocation gauge theory (see [17, 20]) and in the so-called relaxed micromorphic elasticity (see [26]). Moreover, the decomposition of the dislocation density tensor in its three irreducible tensor pieces (called 'tentor', 'trator', 'axitor') with respect to the three-

dimensional orthogonal group is given by Lazar [17] and Lazar and Anastassidis [20]. The corresponding decomposition of $F_{[i j] k}$ is given in Appendix D.

\subsection{Decomposition of the strain gradient tensor}

Now we construct the $O(n)$-irreducible pieces of the strain gradient tensor $\eta_{i j k}$ using the method of Young tableaux. The strain gradient tensor, by definition, is symmetric in its first pair of indices. Thus, it is not a general tensor of rank three $\left(\eta_{i j k} \equiv F_{(i j) k}\right)$.

For the strain gradient tensor there are only two non-vanishing Young tableaux if $n>2$, namely

$$
\begin{aligned}
& \begin{array}{|l|l|l|l|l|l|l|l|l|}
\hline i & j & j \\
\hline k & \\
\hline
\end{array} \\
& \eta_{(i j) k}=\eta_{(i j k)}+\frac{4}{3} \eta_{(j[i) k]}
\end{aligned}
$$

\footnotetext{
${ }^{2}$ Although the correct symmetry operators (Eqs. (4.9) and (4.10) in [30]) are used by Toupin [30], the tensors ${ }_{\mathrm{A}} a_{p q r}$ and ${ }_{\overline{\mathrm{P}}} a_{p q r}$ in Eq. (4.11) in [30] contain misprints. Moreover, the tensor decomposition based on Young tableaux given in this paper is in agreement with the decomposition given by Wade and Bruck [31], namely: ${ }_{\mathrm{S}} F_{i j k} \equiv S_{i j k},{ }_{\mathrm{A}} F_{i j k} \equiv Q_{i j k},{ }_{\mathrm{P}} F_{i j k} \equiv Q_{i j k}^{(1)},{ }_{\mathrm{P}} F_{i j k} \equiv Q_{i j k}^{(2)}$.
} 
with the two tensor pieces

$$
\begin{aligned}
\mathrm{s} \eta_{i j k}:=\eta_{(i j k)} & =\frac{1}{3}\left(\eta_{i j k}+\eta_{j k i}+\eta_{k i j}\right) \\
\overline{\mathrm{P}} \eta_{i j k}:=\frac{4}{3} \eta_{(j[i) k]} & =\frac{1}{3}\left(2 \eta_{i j k}-\eta_{j k i}-\eta_{k i j}\right)
\end{aligned}
$$

which are irreducible with respect to $G L(n)$ and reducible with respect to $O(n)$.

In order to construct the $O(n)$-irreducible pieces of $\eta_{i j k}$, we split the strain gradient tensor $\eta_{i j k}$ into two parts

$$
\eta_{i j k}=\not \nearrow_{i j k}+\frac{1}{n} \delta_{i j} \eta_{\cdot \cdot k}
$$

namely the shear gradient tensor

$$
\not \nearrow_{i j k}:=\eta_{i j k}-\frac{1}{n} \delta_{i j} \eta_{. \cdot k}=\not_{i j, k}
$$

with the two traces

$$
\begin{aligned}
& \not \lambda_{\cdot . k}=0 \\
& \not \lambda_{i . .}=\eta_{i \cdot .}-\frac{1}{n} \eta_{\cdot \cdot i}=\not_{i j, j}
\end{aligned}
$$

and the dilatation gradient vector

$$
\eta_{. . k}:=\eta_{i i k}=\delta_{i j} \eta_{i j k}=\varepsilon_{. ., k}
$$

which is nothing but the gradient of the dilatation $\varepsilon \ldots$ Eq. (23) is the non-vanishing trace of the shear gradient tensor. In this formulation, the vector $\not \nearrow_{i}$. in Eq. (23) is the vector part of the shear gradient tensor and identically the divergence of the shear tensor. Therefore, we call this vector part (23) the shear divergence vector. The dilatation gradient vector cannot be reduced any further, since it is already an irreducible piece, and the application of the Young tableau method to the shear gradient tensor together with the taking of traces yields three further irreducible pieces, provided $n>2$.

Therefore, the strain gradient tensor $\eta_{i j k}$ can be decomposed into four irreducible pieces under the orthogonal group $O(n)$ according to

$$
\eta_{i j k}={ }^{(1)} \eta_{i j k}+{ }^{(2)} \eta_{i j k}+{ }^{(3)} \eta_{i j k}+{ }^{(4)} \eta_{i j k}
$$

possessing the number of independent tensor components

$$
\frac{n^{2}(n+1)}{2}=\frac{n(n-1)(n+4)}{6}+\frac{n\left(n^{2}-4\right)}{3}+n+n
$$

These pieces are invariant under the action of $O(n)$ and $S O(n)$. The irreducible pieces are given in the following.

\subsubsection{The irreducible piece: ${ }^{(1)} \eta_{i j k}$}

The totally symmetric and traceless tensor of rank three is characterized by the following Young tableau: 


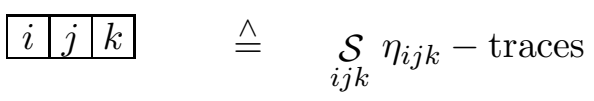

where $\underset{i j k}{\mathcal{S}}$ means the total symmetrization in the indices $i, j, k$ and divided by 3 !. Since the Young symmetry and the trace subtraction are 'commutative' for the construction of $O(n)$-irreducible tensors [13], we obtain

$$
{ }^{(1)} \eta_{i j k}=\eta_{(i j k)}-\text { traces }=\stackrel{\circ}{\eta}_{(i j k)} .
$$

Substituting the traceless tensor of rank three (B.4) into Eq. (27) and doing some algebra, we find

$$
{ }^{(1)} \eta_{i j k}=\frac{1}{3}\left(\eta_{i j k}+\eta_{j k i}+\eta_{k i j}-\frac{1}{n+2}\left(\delta_{i j}\left[2 \eta_{k . .}+\eta_{. \cdot k}\right]+\delta_{i k}\left[2 \eta_{j . .}+\eta_{\cdot . \cdot j}\right]+\delta_{j k}\left[2 \eta_{i . .}+\eta_{\cdot . i}\right]\right)\right) .
$$

It is remarkable that the irreducible piece ${ }^{(1)} \eta_{i j k}$ can be equivalently expressed in terms of the shear gradient tensor and the non-vanishing vector trace of the shear gradient tensor, which is the shear divergence vector, according to

$$
{ }^{(1)} \eta_{i j k}=\frac{1}{3}\left(\lambda_{i j k}+\not_{j k i}+\not_{k i j}-\frac{2}{n+2}\left(\delta_{i j} \lambda_{k . .}+\delta_{i k} \lambda_{j .} .+\delta_{j k} \lambda_{i . .}\right)\right) .
$$

Thus, the piece (29) is the totally symmetric and traceless shear gradient tensor of Young pattern (3) (traceless tensor of symmetry (3)).

\subsubsection{The irreducible piece: ${ }^{(2)} \eta_{i j k}$}

Another irreducible tensor part of $\eta_{i j k}$ is characterized by a traceless tensor of rank three having the following Young symmetry:

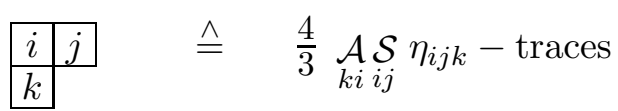

where $\underset{i j}{\mathcal{S}}$ means the symmetrization in the indices $i, j$ and divided by 2 , and $\underset{k i}{\mathcal{A}}$ means the antisymmetrization in the indices $k, i$ and divided by 2 . The factor $4 / 3$ is the normalization factor for the tensor decomposition (17). Using that the Young symmetry and the trace subtraction are 'commutative' operations for the construction of $O(n)$-irreducible tensors [13], we get

$$
{ }^{(2)} \eta_{i j k}=\frac{4}{3} \eta_{(j[i) k]}-\text { traces }=\frac{4}{3} \stackrel{\circ}{\eta(j[i) k]}
$$

Therefore, the tensor ${ }^{(2)} \eta_{i j k}$ is symmetric in the indices $i$ and $j$, and antisymmetric in the indices $i$ and $k$. Substituting the traceless tensor of rank three (B.4) into Eq. (30) and doing some algebra, we obtain

$$
{ }^{(2)} \eta_{i j k}=\frac{1}{3}\left(2 \eta_{i j k}-\eta_{j k i}-\eta_{k i j}+\frac{1}{n-1}\left(2 \delta_{i j}\left[\eta_{k . .}-\eta_{. . .}\right]-\delta_{i k}\left[\eta_{j . .}-\eta_{. . j}\right]-\delta_{j k}\left[\eta_{i . .}-\eta_{. . i}\right]\right)\right) .
$$


Note that the irreducible piece ${ }^{(2)} \eta_{i j k}$ can be equivalently expressed in terms of the shear gradient tensor and the shear divergence vector according to

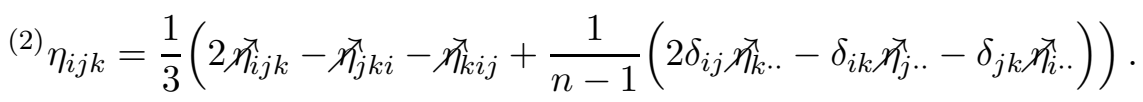

Thus, the piece (32) is the traceless shear gradient tensor with mixed symmetry of Young pattern $(2,1)$ (traceless tensor of symmetry $(2,1)$ ).

\subsubsection{The irreducible pieces: ${ }^{(3)} \eta_{i j k}$ and ${ }^{(4)} \eta_{i j k}$}

There are two irreducible vector pieces corresponding to the two canonical traces, namely the dilatation gradient vector $\eta_{. . k}$ and the shear divergence vector $\not_{i} .$. . The dilatation gradient vector gives the irreducible piece ${ }^{(4)} \eta_{i j k}$ :

$$
{ }^{(4)} \eta_{i j k}=\frac{1}{n} \delta_{i j} \eta_{. . k} .
$$

Using the irreducible decomposition, the condition of incompressibility can be stated as

$$
\varepsilon . .=0, \quad \eta_{. . k}=0, \quad{ }^{(4)} \eta_{i j k}=0 .
$$

The other irreducible vector piece is defined by

$$
{ }^{(3)} \eta_{i j k}=\eta_{i j k}-{ }^{(1)} \eta_{i j k}-{ }^{(2)} \eta_{i j k}-{ }^{(4)} \eta_{i j k}
$$

and reads

$$
\left.{ }^{(3)} \eta_{i j k}=\frac{n}{(n+2)(n-1)}\left(\delta_{i k}\left[\eta_{j . .}-\frac{1}{n} \eta_{. \cdot j}\right]+\delta_{j k}\left[\eta_{i . .}-\frac{1}{n} \eta_{. . \cdot i}\right]-\frac{2}{n} \delta_{i j}\left[\eta_{k . .}-\frac{1}{n} \eta_{. . k}\right]\right)\right) .
$$

The irreducible piece ${ }^{(3)} \eta_{i j k}$ can be completely expressed in terms of the shear divergence vector according to

$$
{ }^{(3)} \eta_{i j k}=\frac{n}{(n+2)(n-1)}\left(\delta_{i k} \nearrow_{j . .}+\delta_{j k} \not \nearrow_{i .}-\frac{2}{n} \delta_{i j} \lambda_{k} . .\right) .
$$

It can be seen that the irreducible piece given in Eq. (37) is the sum of the traces subtracted out in the pieces ${ }^{(1)} \eta_{i j k}$ in Eq. (29) and ${ }^{(2)} \eta_{i j k}$ in Eq. (32).

\subsection{Properties of the irreducible pieces}

First of all, we observe that the presented decomposition of the strain gradient tensor $\eta_{i j k}$ is canonical and unique, since we started from the initial decomposition (20) into the shear gradient tensor and the dilatation gradient vector, whereas the latter is already irreducible. The initial decomposition (20) has ensured that the irreducible subspaces which we have obtained are uniquely defined. The unique decomposition of the shear gradient tensor into its three irreducible pieces is given by

$$
\not_{i j k}={ }^{(1)} \eta_{i j k}+{ }^{(2)} \eta_{i j k}+{ }^{(3)} \eta_{i j k}
$$

which are given by Eqs. (29), (32) and (37). 
There are two types of vanishing traces

$$
\begin{aligned}
& { }^{(1)} \eta_{. . k}={ }^{(2)} \eta_{. \cdot k}={ }^{(3)} \eta_{. . k}=0 \\
& { }^{(1)} \eta_{i . .}={ }^{(2)} \eta_{i . .}=0
\end{aligned}
$$

and the Young symmetries of those parts vanish are given by

$$
{ }^{(1)} \eta_{i[j k]}=0, \quad{ }^{(2)} \eta_{(i j k)}=0 .
$$

There are two types of non-vanishing traces

$$
\begin{aligned}
{ }^{(3)} \eta_{i . .} & =\not \nearrow_{i . .} \\
{ }^{(4)} \eta_{i . .} & =\frac{1}{n} \eta_{. \cdot i} \\
{ }^{(4)} \eta_{. \cdot k} & =\eta_{. \cdot k} .
\end{aligned}
$$

The four irreducible components defined by Eqs. (29), (32), (333) and (37) have an interesting orthogonality property

$$
\begin{array}{ll}
{ }^{(I)} \eta_{i j k}{ }^{(J)} \eta_{i j k}=0, & I \neq J \\
{ }^{(I)} \eta_{i j k}{ }^{(J)} \eta_{i j k} \neq 0, & I=J
\end{array}
$$

so that

$$
\eta_{i j k} \eta_{i j k}=\sum_{I=1}^{4}{ }^{(I)} \eta_{i j k}{ }^{(I)} \eta_{i j k} .
$$

On the other hand, it holds one cross term relation between ${ }^{(3)} \eta_{i j k}$ and ${ }^{(4)} \eta_{k j i}$

$$
{ }^{(3)} \eta_{i j k}{ }^{(4)} \eta_{k j i}=\frac{1}{n} \not \lambda_{i . .} \eta_{. \cdot i}
$$

describing the coupling between the shear divergence vector $\not \nearrow_{i}$. and the dilatation gradient vector $\eta_{\cdot . i}$.

\subsection{Three dimensions: $n=3$}

In three dimensions, the irreducible pieces of $\eta_{i j k}$ are given by 3

$$
\begin{aligned}
\eta_{i j k} & ={ }^{(1)} \eta_{i j k}+{ }^{(2)} \eta_{i j k}+{ }^{(3)} \eta_{i j k}+{ }^{(4)} \eta_{i j k} \\
18 & =7+5+3+3
\end{aligned}
$$

\footnotetext{
${ }^{3}$ In three dimensions, the irreducible tensor pieces ${ }^{(1)} \eta_{i j k},{ }^{(2)} \eta_{i j k},{ }^{(3)} \eta_{i j k},{ }^{(4)} \eta_{i j k}$ have a spin $j=3,2,1,1$, respectively. The spin $j$ is the highest weight which characterizes irreducible representations up to equivalence (see, e.g., [28]).
} 
where 4

$$
\begin{aligned}
& { }^{(1)} \eta_{i j k}=\frac{1}{3}\left(\lambda_{i j k}+\not_{j k i}+\lambda_{k i j}-\frac{2}{5}\left(\delta_{i j} \lambda_{k . .}+\delta_{i k} \not_{j . .}+\delta_{j k} \lambda_{i . .}\right)\right)
\end{aligned}
$$

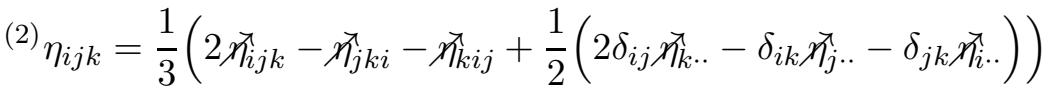

$$
\begin{aligned}
& { }^{(3)} \eta_{i j k}=\frac{3}{10}\left(\delta_{i k} \nearrow_{j} . .+\delta_{j k} \nearrow_{i .} .-\frac{2}{3} \delta_{i j} \lambda_{k} . .\right) \\
& { }^{(4)} \eta_{i j k}=\frac{1}{3} \delta_{i j} \eta_{. \cdot k} \text {. }
\end{aligned}
$$

\subsection{Two dimensions: $n=2$}

In two dimensions, ${ }^{(2)} \eta_{i j k}$ drops out leaving three irreducible pieces due to the theorem that traceless tensors corresponding to Young tableaux in which the sum of the lengths of the first two columns exceeds $n=2$ must be identically zero [13].

Thus, the irreducible pieces of $\eta_{i j k}$ are given by

$$
\begin{aligned}
\eta_{i j k} & ={ }^{(1)} \eta_{i j k}+{ }^{(3)} \eta_{i j k}+{ }^{(4)} \eta_{i j k} \\
6 & =2+2+2
\end{aligned}
$$

where 5

$$
\begin{aligned}
& { }^{(1)} \eta_{i j k}=\frac{1}{3}\left(\not \nearrow_{i j k}+\not \nearrow_{j k i}+\not \nearrow_{k i j}-\frac{1}{2}\left(\delta_{i j} \not \nearrow_{k} . .+\delta_{i k} \not \nearrow_{j .} .+\delta_{j k} \not \nearrow_{i .}\right)\right) \\
& { }^{(3)} \eta_{i j k}=\frac{1}{2}\left(\delta_{i k} \lambda_{j .} .+\delta_{j k} \not \nearrow_{i .}-\delta_{i j} \lambda_{k} . .\right) \\
& { }^{(4)} \eta_{i j k}=\frac{1}{2} \delta_{i j} \eta_{\cdot \cdot k} \text {. }
\end{aligned}
$$

\section{The strain energy density in terms of irreducible strain and irreducible strain gradient tensors}

The most general quadratic form of the strain energy density of isotropic strain gradient elasticity given in terms of the irreducible strain and irreducible strain gradient tensors is given by

$$
W=\frac{1}{2} \varepsilon_{i j}\left(\sum_{I=1}^{2} c_{I}^{(I)} \varepsilon_{i j}\right)+\frac{1}{2} \eta_{i j k}\left(\sum_{I=1}^{4} b_{I}{ }^{(I)} \eta_{i j k}\right)+b_{5}{ }^{(3)} \eta_{i j k}{ }^{(4)} \eta_{k j i} .
$$

Here, $c_{1}$ and $c_{2}$ are 'elastic constants' and $b_{1}, \ldots, b_{5}$ are five 'irreducible gradient parameters' due to the irreducible pieces. The gradient parameters $b_{1}, \ldots, b_{4}$ are the 'coupling constants' for the four irreducible pieces of the strain gradient tensor ${ }^{(1)} \eta_{i j k}, \ldots,{ }^{(4)} \eta_{i j k}$. In particular, $b_{4}$ is the 'coupling constant' for the dilatation gradient vector $\eta \cdot . k$, and $b_{1}, b_{2}, b_{3}$ are the 'coupling

\footnotetext{
${ }^{4}$ Note that the tensor decompositions given in [1, 25] are not canonical since the tensors $S_{1}(\boldsymbol{a})_{i j k}$ and $S_{4}(\boldsymbol{a})_{i j k}$ in [25] and the tensors $T\left(\mathrm{~V}^{\nabla \mathrm{str}}\right)_{i j k}$, and $T\left(\mathrm{~V}^{\nabla \text { rot }}\right)_{i j k}$ in [1] are arbitrary combinations of the irreducible pieces ${ }^{(3)} \eta_{i j k}$ and ${ }^{(4)} \eta_{i j k}$.

${ }^{5}$ The three irreducible pieces ${ }^{(1)} \eta_{i j k},{ }^{(3)} \eta_{i j k}$, and ${ }^{(4)} \eta_{i j k}$ correspond to the three tensors $H_{(i j k)}, T\left(\mathrm{~V}^{\nabla \mathrm{dev}}\right)_{i j k}$, and $T\left(\mathrm{~V}^{\nabla \mathrm{sph}}\right)_{i j k}$ given in [2].
} 
constants' for the three irreducible pieces of the shear gradient tensor ${ }^{(1)} \eta_{i j k},{ }^{(2)} \eta_{i j k},{ }^{(3)} \eta_{i j k}$, respectively. Note the peculiar cross term with gradient parameter $b_{5}$

$$
{ }^{(3)} \eta_{i j k}{ }^{(4)} \eta_{k j i}=\frac{1}{n} \not \eta_{i . .} \eta_{. \cdot i}=\frac{1}{n} \eta_{i . .} \eta_{. . i}-\frac{1}{n^{2}} \eta_{. . i} \eta_{. \cdot i} .
$$

In fact, the gradient parameter $b_{5}$ is the 'coupling constant' between the shear divergence vector $\nearrow_{i .}$. and the dilatation gradient vector $\eta_{. . i}$. Also, notice that since for $n=2$ the piece

${ }^{(2)} \eta_{i j k}$ drops out, there are only four gradient parameters: $b_{1}, b_{3}, b_{4}, b_{5}$ in two-dimensional isotropic strain gradient elasticity.

We can connect the constants $c_{1}$ and $c_{2}$ with the moduli known from elasticity theory

$$
c_{1}=2 \mu, \quad c_{2}=n K, \quad K=\lambda+\frac{2}{n} \mu
$$

where $\mu$ and $\lambda$ are the Lamé constants and $K$ is the modulus of compression or bulk modulus (see, e.g., [16]) for $n$-dimensions.

Using Eq. (60), we define the conjugate quantities of isotropic strain gradient elasticity, namely the Cauchy stress tensor is given by

$$
\sigma_{i j}:=\frac{\partial W}{\partial \varepsilon_{i j}}=\sum_{I=1}^{2} c_{I}^{(I)} \varepsilon_{i j}=c_{1} \not_{i j}+\frac{c_{2}}{n} \delta_{i j} \varepsilon
$$

and the double stress tensor is given by

$$
\tau_{i j k}:=\frac{\partial W}{\partial \eta_{i j k}}=\sum_{I=1}^{4} b_{I}^{(I)} \eta_{i j k}+\frac{b_{5}}{2 n}\left(\delta_{i k} \eta_{l l j}+\delta_{j k} \eta_{l l i}+2 \delta_{i j} \eta_{k l l}\right)-\frac{2 b_{5}}{n^{2}} \delta_{i j} \eta_{l l k}
$$

By means of setting $b_{5}=0$, we 'switch off' the coupling between the shear divergence vector and the dilatation gradient vector and obtain the double stress tensor in terms of the four irreducible pieces and the corresponding four gradient parameters

$$
\tau_{i j k}=\sum_{I=1}^{4} b_{I}^{(I)} \eta_{i j k}
$$

In strain gradient elasticity, the equilibrium condition reads

$$
\sigma_{i j, j}-\tau_{i j k, k j}+f_{i}=0
$$

where $f_{i}$ is the body force density vector.

\section{Relation to Mindlin's strain gradient elasticity of form II}

\subsection{The general case: $n \geq 3$}

The strain energy density of Mindlin's isotropic strain gradient elasticity theory of form II reads [23] (see also [24, 29])

$$
W=\frac{1}{2} \lambda \varepsilon_{i i} \varepsilon_{j j}+\mu \varepsilon_{i j} \varepsilon_{i j}+a_{1} \eta_{i i k} \eta_{k j j}+a_{2} \eta_{i i k} \eta_{j j k}+a_{3} \eta_{i j j} \eta_{i k k}+a_{4} \eta_{i j k} \eta_{i j k}+a_{5} \eta_{i j k} \eta_{k j i}
$$


where $a_{1}, a_{2}, a_{3}, a_{4}, a_{5}$ are the Mindlin gradient parameters in Mindlin's first strain gradient elasticity theory.

Now the Cauchy stress tensor reads

$$
\sigma_{i j}=2 \mu \varepsilon_{i j}+\lambda \delta_{i j} \varepsilon
$$

and the double stress tensor is given by

$\tau_{i j k}=\frac{a_{1}}{2}\left(\delta_{i k} \eta_{l l j}+\delta_{j k} \eta_{l l i}+2 \delta_{i j} \eta_{k l l}\right)+2 a_{2} \delta_{i j} \eta_{l l k}+a_{3}\left(\delta_{i k} \eta_{j l l}+\delta_{j k} \eta_{i l l}\right)+2 a_{4} \eta_{i j k}+a_{5}\left(\eta_{j k i}+\eta_{k i j}\right)$.

Substituting the irreducible pieces (28), (31), (33), and (36) into Eq. (64), and comparing now with Eq. (69), we obtain the relation between Mindlin's gradient parameters and the 'irreducible gradient parameters'

$$
\begin{aligned}
& a_{1}=-\frac{2 b_{1}}{3(n+2)}+\frac{2 b_{2}}{3(n-1)}-\frac{2 b_{3}}{(n+2)(n-1)}+\frac{1}{n} b_{5} \\
& a_{2}=-\frac{b_{1}}{6(n+2)}-\frac{b_{2}}{3(n-1)}+\frac{b_{3}}{(n+2)(n-1) n}+\frac{1}{2 n} b_{4}-\frac{1}{n^{2}} b_{5} \\
& a_{3}=-\frac{2 b_{1}}{3(n+2)}-\frac{b_{2}}{3(n-1)}+\frac{n b_{3}}{(n+2)(n-1)} \\
& a_{4}=\frac{1}{6} b_{1}+\frac{1}{3} b_{2} \\
& a_{5}=\frac{1}{3} b_{1}-\frac{1}{3} b_{2}
\end{aligned}
$$

and the inverse relations between the 'irreducible gradient parameters' and Mindlin's gradient parameters

$$
\begin{aligned}
& b_{1}=2 a_{4}+2 a_{5} \\
& b_{2}=2 a_{4}-a_{5} \\
& b_{3}=\frac{(n+2)(n-1) a_{3}+2 n a_{4}+(n-2) a_{5}}{n} \\
& b_{4}=2 \frac{n a_{1}+n^{2} a_{2}+a_{3}+n a_{4}+a_{5}}{n} \\
& b_{5}=n a_{1}+2 a_{3}+2 a_{5} .
\end{aligned}
$$

If $b_{5}=0$ (no coupling between the shear divergence vector and the dilatation gradient vector), then one parameter of the five Mindlin gradient parameters can be eliminated using the constraint obtained from Eq. (79)

$$
n a_{1}+2 a_{3}+2 a_{5}=0
$$

\subsection{The case: $n=2$}

Because for $n=2$ the piece ${ }^{(2)} \eta_{i j k}$ drops out, there are only four gradient parameters: $b_{1}, b_{3}, b_{4}, b_{5}$ and therefore

$$
b_{2}=0: \quad \Longrightarrow \quad a_{5}=2 a_{4} \text {. }
$$


Using Eq. (81), the strain energy density (67) of Mindlin's isotropic strain gradient elasticity theory of form II reads in two dimensions

$$
W=\frac{1}{2} \lambda \varepsilon_{i i} \varepsilon_{j j}+\mu \varepsilon_{i j} \varepsilon_{i j}+a_{1} \eta_{i i k} \eta_{k j j}+a_{2} \eta_{i i k} \eta_{j j k}+a_{3} \eta_{i j j} \eta_{i k k}+a_{4}\left(\eta_{i j k} \eta_{i j k}+2 \eta_{i j k} \eta_{k j i}\right)
$$

and the double stress tensor (69) is given by

$$
\tau_{i j k}=\frac{a_{1}}{2}\left(\delta_{i k} \eta_{l l j}+\delta_{j k} \eta_{l l i}+2 \delta_{i j} \eta_{k l l}\right)+2 a_{2} \delta_{i j} \eta_{l l k}+a_{3}\left(\delta_{i k} \eta_{j l l}+\delta_{j k} \eta_{i l l}\right)+2 a_{4}\left(\eta_{i j k}+\eta_{j k i}+\eta_{k i j}\right)
$$

in terms of four (two-dimensional) Mindlin gradient parameters $a_{1}, \ldots, a_{4}$.

If $b_{5}=0$, then one parameter of the four Mindlin gradient parameter can be eliminated by substituting Eq. (79) into Eq. (81)

$$
b_{5}=0: \quad \Longrightarrow \quad a_{4}=-\frac{a_{1}+a_{3}}{2} .
$$

\section{Relation to strain gradient elasticity of Helmholtz type}

For $n=3$, a simplified and useful version of strain gradient elasticity is called strain gradient elasticity of Helmholtz type which is a particular case of Mindlin's strain gradient elasticity of form II (see, e.g., [18, 19, 21, 22]). The connection between the strain energy density of isotropic strain gradient elasticity given in terms of the irreducible strain and the strain energy density of strain gradient elasticity of Helmholtz type is given by the particular choice of the gradient parameters

$$
b_{1}=b_{2}=b_{3}=\ell^{2} c_{1}, \quad b_{4}=\ell^{2} c_{2}, \quad b_{5}=0
$$

where $\ell$ is a length scale parameter, and equivalently in terms of Mindlin's gradient parameters

$$
a_{1}=a_{3}=a_{5}=0, \quad a_{2}=-\frac{\ell^{2} c_{1}}{6}+\frac{\ell^{2} c_{2}}{6}, \quad a_{4}=\frac{\ell^{2} c_{1}}{2} .
$$

Then the double stress tensor (64) reads

$$
\tau_{i j k}=\ell^{2}\left(c_{1} \nearrow_{i j k}+\frac{c_{2}}{3} \delta_{i j} \eta_{. . k}\right)=\ell^{2}\left(2 \mu \eta_{i j k}+\lambda \delta_{i j} \eta_{. \cdot k}\right) .
$$

Thus, gradient elasticity of Helmholtz type is based on the initial decomposition (20) into the shear gradient tensor $\not_{i j k}$ and the dilatation gradient vector $\eta_{. . k}$.

\section{Conclusions}

The irreducible decompositions presented here are of interest in themselves, both from the mechanical and group-theoretical point of view. One main motivation for the present work, based on the well proven relevance in strain gradient theories, is their use in setting up the strain energy density and Lagrangian for isotropic strain gradient theories.

Using group theory, a canonical and unique tensor decomposition of the strain gradient tensor into the $O(n)$-irreducible pieces has been given. We have found that: 
- $n=3$ : the strain gradient tensor can be decomposed into four irreducible tensor pieces with $7+5+3+3$ independent components

- $n=2$ : the strain gradient tensor can be decomposed into three irreducible tensor pieces with 2+2+2 independent components.

The four irreducible pieces are built up from the three parts:

- shear gradient tensor $\not \widehat{X}_{i j k}:{ }^{(1)} \eta_{i j k},{ }^{(2)} \eta_{i j k}$

- shear divergence vector $\not \nearrow_{i . .}:{ }^{(1)} \eta_{i j k},{ }^{(2)} \eta_{i j k},{ }^{(3)} \eta_{i j k}$

- dilatation gradient vector $\eta_{. \cdot k}:{ }^{(4)} \eta_{i j k}$.

The group-theoretical interpretation of the four irreducible pieces under $S O(3)$ is:

- ${ }^{(1)} \eta_{i j k}$ : totally symmetric and traceless shear gradient tensor is spin-3 field

- ${ }^{(2)} \eta_{i j k}$ : traceless shear gradient tensor of Young symmetry $(2,1)$ is spin-2 field

- ${ }^{(3)} \eta_{i j k}$ : shear divergence vector in the subtracted traces of ${ }^{(1)} \eta_{i j k}$ and ${ }^{(2)} \eta_{i j k}$ is spin-1 field

- ${ }^{(4)} \eta_{i j k}$ : gradient of the dilatation is spin-1 field.

As a straightforward consequence of the irreducible tensor decomposition, we found the number of gradient parameters:

- $n=3$ : five gradient parameters

- $n=2$ : four gradient parameters

in addition to the two Lamé constants.

Also we discussed the particular case, $b_{5}=0$, when the coupling between the shear divergence vector and the dilatation gradient vector vanishes.

The presented irreducible decomposition of the strain gradient tensor can be also used in strain gradient plasticity (e.g., [8, 9] ) where usually a decomposition into three tensor pieces is used.

\section{Acknowledgements}

The author gratefully acknowledges grants from the Deutsche Forschungsgemeinschaft (Grant Nos. La1974/3-1 and La1974/3-2). In addition, useful remarks from Rainer Glüge are gratefully acknowledged.

\section{A Young tableaux, Young operators and irreducible tensors}

In this Appendix for the convenience of the reader we collect some facts about Young tableaux and irreducible tensors (see [3, 5, 13, 32, 33]).

An irreducible representation $\Delta^{[m]}$ of the symmetric group $S_{n}$ is uniquely determined by the idempotent (normalized) Young operator

$$
\mathcal{Y}_{[m]}=\frac{f_{[m]}}{n !} \mathcal{Q} \mathcal{P}
$$




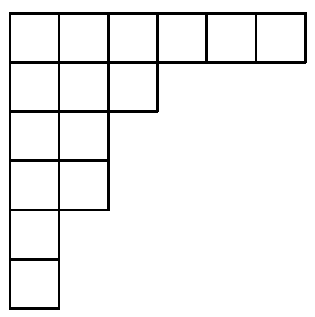

$$
\begin{aligned}
& m_{1} \text { boxes } \\
& m_{2} \text { boxes } \\
& \vdots \\
& m_{r} \text { boxes }
\end{aligned}
$$

Figure 1: Young pattern $\underline{m}$

with

$$
\begin{array}{ll}
\mathcal{P}=\sum_{p \in H_{[m]}} p & \text { 'symmetrizer' } \\
\mathcal{Q}=\sum_{q \in V_{[m]}} \delta_{q} q & \text { 'antisymmetrizer' }
\end{array}
$$

and

$$
\mathcal{Y}_{[m]} \mathcal{Y}_{\left[m^{\prime}\right]}=\delta_{[m]\left[m^{\prime}\right]} \mathcal{Y}_{[m]}
$$

which is related to a Young tableau denoted by $[m]$. Here $\mathcal{P}$ is the operator for horizontal permutations in the diagram and $\mathcal{Q}$ is the operator for vertical permutations. Horizontal permutations $p$ are permutations which interchange only symbols (or indices) in the same row. Vertical permutations $q$ interchange only symbols (or indices) in the same column and $\delta_{q}$ is the parity of the permutation $q$.

If

$$
\underline{m}=\left(m_{1}, m_{2}, \ldots m_{r}\right) \quad \text { with } \quad m_{1} \geq m_{2} \geq \ldots \geq m_{r}, \quad \sum_{i=1}^{r} m_{i}=n
$$

defines a Young pattern (Fig. 1), then a Young tableau $[\mathrm{m}]$ is obtained by putting in (without repetition) the indices $i_{1}, \ldots i_{n}$ of a tensor of rank $n, F_{i_{1} \ldots i_{n}}$, and $H_{[m]}$ and $V_{[m]}$ denotes their horizontal and vertical permutations with respect to $[\mathrm{m}]$. A standard tableau is obtained when the indices $i_{1}, \ldots, i_{n}$ are ordered lexicographically. There are

$$
f_{[m]}=n ! \frac{\prod_{i<j}\left(l_{i}-l_{j}\right)}{\prod_{i=1}^{r} l_{i} !} \quad \text { with } \quad l_{i}=m_{i}+r-i, \quad \sum_{[m]} f_{[m]}^{2}=n !
$$

different standard tableaux which correspond to $f_{[m]}$ different, but equivalent, irreducible representations of $S_{n}$ whose dimension is given also by $f_{[m]}$. The (normalized) Young operators $\mathcal{Y}_{[m]}$ according to Eq. (A.4) project onto mutually orthogonal irreducible right ideals which provide irreducible representations of $S_{n}$.

Thus, in order to obtain a tensor of rank $n$ with the symmetry described by a Young pattern $\underline{m}$, we apply the Young operator $\mathcal{Y}_{[m]}$ to the tensor $F_{i_{1} \ldots i_{n}}$ :

$$
F_{i_{1} \ldots i_{n}}^{[m]}=\mathcal{Y}_{[m]} F_{i_{1} \ldots i_{n}} .
$$

Consequently, the tensor will be symmetric in all the indices which appear in the same row and antisymmetric in all the indices which appear in the same column. Any tensor component for 
which an index appears twice in the same column is equal to zero. A tensor having a certain Young symmetry is irreducible with respect to the general linear group $G L(n)$.

Going from the group $G L(n)$ to the orthogonal group $O(n)$, only the completely antisymmetric tensors remain irreducible. The reason is that, because of the definition of the orthogonal group, $\delta_{i j} a_{i k} a_{j l}=\delta_{k l}, \forall \boldsymbol{a} \in O(n)$, the operation of taking the trace (contraction) of a tensor commutes with the orthogonal transformations of that tensor:

$$
\operatorname{tr} \boldsymbol{F}^{\prime}=\delta_{i j} F_{i j}^{\prime}=\delta_{i j} a_{i k} a_{j l} F_{k l}=\operatorname{tr} \boldsymbol{F} .
$$

By Schur's Lemma, irreducible tensors with respect to the orthogonal group are traceless tensors having definite symmetry class (Young symmetry). This decomposition is obtained as follows:

$$
F_{i_{1} \ldots i_{n}}^{[m]}=\stackrel{\circ}{F}_{i_{1} \ldots i_{n}}^{[m]}+\sum_{1 \leq r, s \leq n} \delta_{i_{r} i_{s}} F_{i_{1} \ldots i_{r-1} i_{r+1} \ldots i_{s-1} i_{s+1} \ldots i_{n}}^{[m-2]} .
$$

The tensors which appear under the sum in Eq. (A.9) have rank $n-2$ and a Young pattern $[m-2] \subset[m]$ obtained by removing two boxes from the pattern. They may be decomposed again into traceless ones plus some remainder, and so on. Therefore, a traceless tensor is obtained from the original one by subtracting all the traces.

In general, there are two possible ways to construct $O(n)$-irreducible tensors. Either one symmetrizes the indices according to the corresponding (standard) Young tableaux and afterwards subtracts all the traces, or one starts from tensors being already traceless and finally symmetrizes because this does not destroy the tracelessness. In this sense, the operations of Young symmetrization and subtraction of traces can be interchanged and are 'commutative'.

\section{B Traceless tensor of rank three}

Here, we decompose a general tensor of rank three $F_{i j k}$ into a traceless tensor of rank three $\stackrel{\circ}{F}_{i j k}$ and the three traces, given in terms of three vectors $H_{k}, K_{j}$ and $L_{i}$, according to (see, e.g., [13])

$$
F_{i j k}=\stackrel{\circ}{F}_{i j k}+\delta_{i j} H_{k}+\delta_{i k} K_{j}+\delta_{j k} L_{i}
$$

We require that the tensor $\stackrel{\circ}{F}_{i j k}$ be traceless and obtain from Eq. (B.1):

$$
\begin{array}{ll}
\delta_{i j}: & F_{. . k}=n H_{k}+K_{k}+L_{k} \\
\delta_{i k}: & F_{. j .}=H_{j}+n K_{j}+L_{j} \\
\delta_{j k}: & F_{i . .}=H_{i}+K_{i}+n L_{i}
\end{array}
$$

where we use the notation $F_{. . k} \equiv F_{i i k}$, and $\delta_{i i}=n$. Solving (B.2), we find

$$
\begin{aligned}
H_{i} & =\frac{1}{(n+2)(n-1)}\left[(n+1) F_{. . i}-F_{. i .}-F_{i . .}\right] \\
K_{i} & =\frac{1}{(n+2)(n-1)}\left[-F_{. . i}+(n+1) F_{. i .}-F_{i . .}\right] \\
L_{i} & =\frac{1}{(n+2)(n-1)}\left[-F_{. . i}-F_{. i .}+(n+1) F_{i . .}\right] .
\end{aligned}
$$


Thus, a traceless tensor of rank three reads

$$
\stackrel{\circ}{F}_{i j k}=F_{i j k}-\delta_{i j} H_{k}-\delta_{i k} K_{j}-\delta_{j k} L_{i}
$$

with Eq. (B.3). The decomposition (B.1) is unique [13, 33].

\section{Irreducible pieces for gradient elasticity of form I}

For completeness and convenience of the reader, we give the $O(n)$-irreducible pieces for gradient elasticity of form I.

In gradient elasticity of form I 23], the tensor of rank three, which is the second gradient of the displacement vector, is used

$$
\eta_{i j k}:=u_{k, i j}
$$

and the two traces read

$$
\eta_{. . k}=u_{k, l l}=\Delta u_{k}
$$

and

$$
\eta_{k . .}=u_{l, l k}
$$

Substituting Eqs. (C.1)-(C.3) into Eqs. (28), (31), (36) and (33), the four $O(n)$-irreducible pieces for gradient elasticity of form I read

$$
\begin{aligned}
{ }^{(1)} \eta_{i j k}= & \frac{1}{3}\left(u_{k, i j}+u_{i, j k}+u_{j, k i}\right. \\
& \left.\quad-\frac{1}{n+2}\left(\delta_{i j}\left[2 u_{l, l k}+u_{k, l l}\right]+\delta_{i k}\left[2 u_{l, l j}+u_{j, l l}\right]+\delta_{j k}\left[2 u_{l, l i}+u_{i, l l}\right]\right)\right) \\
{ }^{(2)} \eta_{i j k}= & \frac{1}{3}\left(2 u_{k, i j}-u_{i, j k}-u_{j, k i}\right. \\
& \left.\quad+\frac{1}{n-1}\left(2 \delta_{i j}\left[u_{l, l k}-u_{k, l l}\right]-\delta_{i k}\left[u_{l, l j}-u_{j, l l}\right]-\delta_{j k}\left[u_{l, l i}-u_{i, l l}\right]\right)\right) \\
{ }^{(3)} \eta_{i j k}= & \left.\frac{n}{(n+2)(n-1)}\left(\delta_{i k}\left[u_{l, l j}-\frac{1}{n} u_{j, l l}\right]+\delta_{j k}\left[u_{l, l i}-\frac{1}{n} u_{i, l l}\right]-\frac{2}{n} \delta_{i j}\left[u_{l, l k}-\frac{1}{n} u_{k, l l}\right]\right)\right) \\
{ }^{(4)} \eta_{i j k}= & \frac{1}{n} \delta_{i j} u_{k, l l} .
\end{aligned}
$$

\section{Irreducible decomposition of the tensor $F_{[i j] k}$}

We now give the decomposition of the tensor $\kappa_{i j k}:=F_{[i j] k}\left(\kappa_{i j k}=-\kappa_{j i k}\right)$ into its $O(n)$ irreducible pieces.

For the tensor $\kappa_{i j k}$ there are only two non-vanishing Young tableaux if $n>2$, namely

$$
\begin{array}{l|l|l|}
\hline i \\
\hline j
\end{array} \otimes k=\beta \begin{array}{l|l|}
\hline i \\
\hline j & k \\
\hline j \\
\hline k \\
\hline
\end{array}
$$




$$
\kappa_{[i j] k}=\frac{4}{3} \kappa_{[j(i] k)}+\kappa_{[i j k]}
$$

with the two tensor pieces

$$
\begin{gathered}
\mathrm{P} \kappa_{i j k}:=\frac{4}{3} \kappa_{[j(i] k)}=\frac{1}{3}\left(2 \kappa_{i j k}-\kappa_{j k i}-\kappa_{k i j}\right) \\
{ }_{\mathrm{A}} \kappa_{i j k}:=\kappa_{[i j k]}=\frac{1}{3}\left(\kappa_{i j k}+\kappa_{j k i}+\kappa_{k i j}\right)
\end{gathered}
$$

which are irreducible with respect to $G L(n)$. Moreover, ${ }_{\mathrm{A}} \kappa_{i j k}$ is already irreducible and ${ }_{\mathrm{P}} \kappa_{i j k}$ is reducible with respect to $O(n)$. Note that Toupin [30] called ${ }_{\mathrm{P}} \kappa_{i j k}$ the principal part.

Finally, the tensor $\kappa_{i j k}$ can be decomposed into three irreducible pieces under the orthogonal group $O(n)$ if we split the principal part $\mathrm{p} \kappa_{i j k}$ into its traceless and trace parts. For $n>2$, we may write its three irreducible pieces according to

$$
\kappa_{i j k}={ }^{(1)} \kappa_{i j k}+{ }^{(2)} \kappa_{i j k}+{ }^{(3)} \kappa_{i j k}
$$

with the number of independent tensor components

$$
\frac{n^{2}(n-1)}{2}=\frac{n\left(n^{2}-4\right)}{3}+n+\frac{n(n-1)(n-2)}{6}
$$

and the three $O(n)$-irreducible pieces are given by

$$
\begin{aligned}
& { }^{(1)} \kappa_{i j k}:={ }_{\mathrm{P}} \stackrel{\circ}{i j k}_{i j k} \equiv \frac{4}{3} \stackrel{\circ}{\kappa}_{[j(i] k)}=\frac{1}{3}\left(2 \kappa_{i j k}-\kappa_{j k i}-\kappa_{k i j}\right)+\frac{1}{n-1}\left(\delta_{i k} \kappa_{j . .}-\delta_{j k} \kappa_{i . .}\right) \\
& { }^{(2)} \kappa_{i j k}=\frac{1}{n-1}\left(\delta_{j k} \kappa_{i . .}-\delta_{i k} \kappa_{j . .}\right) \\
& { }^{(3)} \kappa_{i j k}:={ }_{\mathrm{A}} \kappa_{i j k}=\frac{1}{3}\left(\kappa_{i j k}+\kappa_{j k i}+\kappa_{k i j}\right) .
\end{aligned}
$$

The irreducible pieces are canonical. Under the group $S O(3)$, the pieces ${ }^{(1)} \kappa_{i j k},{ }^{(2)} \kappa_{i j k},{ }^{(3)} \kappa_{i j k}$ are spin-2, spin-1, spin-0 fields, respectively. For $n=2$, the tensor $\kappa_{i j k}$ is already irreducible.

For $n=3$, we may define the dual tensor of $\kappa_{i j k}$ according to

$$
\kappa_{i j}:=\frac{1}{2} \epsilon_{k l j} \kappa_{k l i}
$$

and the inverse relation reads

$$
\kappa_{j k i}=\epsilon_{j k n} \kappa_{i n}
$$

For the irreducible pieces, it holds for every irreducible piece: ${ }^{(I)} \kappa_{i j}=\frac{1}{2} \epsilon_{k l j}{ }^{(I)} \kappa_{k l i}$ for $I=$ $1,2,3$. Then the three dual $O(3)$-irreducible pieces are

$$
\kappa_{i j}={ }^{(1)} \kappa_{i j}+{ }^{(2)} \kappa_{i j}+{ }^{(3)} \kappa_{i j}
$$

where

$$
\begin{aligned}
& { }^{(1)} \kappa_{i j}=\kappa_{(i j)}-\frac{1}{3} \delta_{i j} \kappa_{l l}=\widehat{h}_{(i j)} \\
& { }^{(2)} \kappa_{i j}=\kappa_{[i j]} \\
& { }^{(3)} \kappa_{i j}=\frac{1}{3} \delta_{i j} \kappa_{l l} .
\end{aligned}
$$

Thus, ${ }^{(1)} \kappa_{i j}$ is the symmetric and traceless part (or symmetric deviator) of $\kappa_{i j},{ }^{(2)} \kappa_{i j}$ is the antisymmetric part of $\kappa_{i j}$, and ${ }^{(3)} \kappa_{i j}$ is the trace part (or spherical part) of $\kappa_{i j}$. The pieces ${ }^{(1)} \kappa_{i j},{ }^{(2)} \kappa_{i j},{ }^{(3)} \kappa_{i j}$ are spin-2, spin-1, spin-0 fields, respectively. 


\section{E Irreducible decomposition of a general tensor of rank three $F_{i j k}$}

As an important side-result of this paper, we give the irreducible decomposition of a general tensor of rank three $F_{i j k}$ with respect to $O(n)$. The first step is to decompose the general tensor $F_{i j k}$ in the indices $i$ and $j$ into symmetric and antisymmetric parts. In the second step, we use the irreducible decomposition of these two tensors. For $n>2$, a general tensor of rank three, $F_{i j k}$, can be uniquely decomposed into seven $O(n)$-irreducible pieces according to

$$
\begin{aligned}
F_{i j k} & =F_{(i j) k}+F_{[i j] k}=\eta_{i j k}+\kappa_{i j k} \\
& ={ }^{(1)} \eta_{i j k}+{ }^{(2)} \eta_{i j k}+{ }^{(3)} \eta_{i j k}+{ }^{(4)} \eta_{i j k}+{ }^{(1)} \kappa_{i j k}+{ }^{(2)} \kappa_{i j k}+{ }^{(3)} \kappa_{i j k}
\end{aligned}
$$

with the seven irreducible tensor pieces given by Eqs. (29), (32), (37), (33), (D.7), (D.8), (D.9). For instance, the irreducible decomposition (E.1) can be used in micromorphic elasticity for the micro-deformation gradient tensor (see, e.g., [23, 7]).

\section{References}

[1] N. Auffray, On the algebraic structure of isotropic generalized elasticity theories, Mathematics and Mechanics of Solids 20, 565-581 (2015).

[2] N. Auffray, On the isotropic moduli of 2D strain-gradient elasticity, Continuum Mechanics and Thermodynamics 27, 5-19 (2015).

[3] A.O. Barut and R. Raczka, Theory of Group Representations and Applications, PWN Polish Scientific Publishers, Warszawa (1977).

[4] A. Bertram, Elasticity and Plasticity of Large Deformations, Springer, Berlin (2005).

[5] H. Boerner, Darstellungen von Gruppen, Springer-Verlag, Berlin (1967); Representations of Groups, North-Holland, Amsterdam (1970).

[6] P. Chadwick, Continuum Mechanics: Concise Theory and Problems, George Allen and Unwin, London (1976).

[7] A.C. Eringen, Microcontinuum Field Theories I: Foundations and Solids, Springer, New York (1999).

[8] N.A. Fleck and J.W. Hutchinson, Strain gradient plasticity, Advances in Applied Mechanics 33, 296-361 (1997).

[9] H. Gao, Y. Huang, W.D. Nix, and J.W. Hutchinson, Mechanism-based strain gradient plasticity - I. Theory, Journal of the Mechanics and Physics of Solids 47, 1239-1263 (1999).

[10] B. Geyer, M. Lazar, and D. Robaschik, Decomposition of non-local light-cone operators into harmonic operators of definite twist, Nuclear Physics B 559, 339-377 (1999).

[11] B. Geyer and M. Lazar, Twist decomposition of nonlocal light-cone operators II: general tensors of 2nd rank, Nuclear Physics B 581, 341-390 (2000).

[12] R. Glüge, J. Kalisch, and A. Bertram, The eigenmodes in isotropic strain gradient elasticity, in: Generalized Continua as Models for Classical and Advanced Materials, pp. 163-178, Eds.: H. Altenbach and S. Forest, Springer (2016). 
[13] M. Hamermesh, Group Theory and Its Application to Physical Problems, AddisonWesley, London (1962); Dover, New York (1989).

[14] F.W. Hehl, J.D. McCrea, E.W. Mielke, and Y. Ne'eman, Metric-affine gauge theory of gravity: Field equations, Noether identities, world spinors, and breaking of dilation invariance, Physics Reports 258, 1-171 (1995).

[15] Y. Itin and F.W. Hehl, Irreducible decompositions of the elasticity tensor under the linear and orthogonal groups and their physical consequences, Journal of Physics: Conference Series 597, 012046 (2015).

[16] L.D. Landau and E.M. Lifschitz, Theory of Elasticity: Volume 7 (Course of Theoretical Physics), Pergamon Press Ltd., Oxford (1970).

[17] M. Lazar, An elastoplastic theory of dislocations as a physical field theory with torsion, Journal of Physics A: Mathematical and General 35, 1983-2004 (2002).

[18] M. Lazar and G.A. Maugin, Nonsingular stress and strain fields of dislocations and disclinations in first strain gradient elasticity, International Journal of Engineering Science 43, 1157-1184 (2005).

[19] M. Lazar, G.A. Maugin, and E.C. Aifantis, On dislocations in a special class of generalized elasticity, physica status solidi (b) 242, 2365-2390 (2005).

[20] M. Lazar and C. Anastassiadis, Gauge theory of dislocations: static solutions of screw and edge dislocations, Philosophical Magazine 89, 199-231 (2009).

[21] M. Lazar, The fundamentals of non-singular dislocations in the theory of gradient elasticity: Dislocation loops and straight dislocations, International Journal of Solids and Structures 50, 352-362 (2013).

[22] M. Lazar, On gradient field theories: gradient magnetostatics and gradient elasticity, Philosophical Magazine 94, 2840-2874 (2014).

[23] R.D. Mindlin, Micro-structure in linear elasticity, Archive for Rational Mechanics and Analysis 16, 51-78 (1964).

[24] R.D. Mindlin and N.N. Eshel, On first strain-gradient theories in linear elasticity, International Journal of Solids and Structures 4, 109-124 (1968).

[25] V. Monchiet and G. Bonnet, Inversion of higher order isotropic tensors with minor symmetries and solution of higher order heterogeneity problems, Proceedings of the Royal Society A 467, 314-332 (2011).

[26] P. Neff, I.-D. Ghiba, M. Lazar, and A. Madeo, The relaxed linear micromorphic continuum: well-posedness of the static problem and relations to the gauge theory of dislocations, The Quarterly Journal of Mechanics and Applied Mathematics 68, 53-84 (2015).

[27] J.A. Schouten, Tensor Analysis for Physicits, Oxford University Press, Oxford (1951).

[28] R.U. Sexl and H.K. Urbantke, Relativity, Groups, Particles: Special Relativity and Relativistic Symmetry in Field and Particle Physics, Springer, Wien (2001). 
[29] H.M. Shodja, A. Zaheri, and A. Tehranchi, Ab initio calculations of characteristic lengths of crystalline materials in first strain gradient elasticity, Mechanics of Materials 61, 73-78 (2013).

[30] R.A. Toupin, Elastic materials with couple-stresses, Archive for Rational Mechanics and Analysis 11, 385-414 (1962).

[31] T.L. Wade and R.H. Bruck, Types of Symmetries, The American Mathematical Monthly 51, 123-129 (1944).

[32] H. Weyl, Gruppentheorie und Quantenmechanik, S. Hirzel, Leipig (1931); The Group Theory and Quantum Mechanics, Dover, New York (1955).

[33] H. Weyl, The Classical Groups: Their Invariants and Representations, Princeton University Press Princeton (1946). 